\title{
Toxicological Assessment of Silver Nanoparticles Synthesized through Green Route using Andrographis paniculata
}

\author{
Soumendra Darbar ${ }^{1,2}$, Srimoyee Saha3 ${ }^{3}$ Kausikisankar Pramanik², Atiskumar Chattopadhyay1,* \\ ${ }^{1}$ Faculty of Science, Jadavpur University, Raja S C Mallick Road, Kolkata - 70 0032, West Bengal, India. \\ ${ }^{2}$ Department of Chemistry, Jadavpur University, Raja S C Mallick Road, Kolkata - 700 032, West Bengal, India. \\ ${ }^{3}$ Department of Physics, Jadavpur University, Raja S C Mallick Road, Kolkata - 700 032, West Bengal, India.
}

\section{ART I C LE DETAILS}

\section{Article history:}

Received 12 October 2018

Accepted 28 November 2018

Available online 16 March 2019

\section{Keywords:}

Acute Toxicity

Nanomedicine

Silver Nanoparticles

Andrographis paniculata

Mice Model

\begin{abstract}
A B S T R A C T
Nanomedicine is a combination of nanotechnology and medicine. It provides new direction in medical diagnosis, monitoring and treatment at the level of single molecules or molecular assemblies at the "nano" scale. Additionally, continuing improvement in the pharmacological and therapeutic properties of drugs is driving the revolution in novel drug delivery systems. Nanomedicine is widely explored nowadays for treatment of life threatening diseases, yet comes with various challenges and questions. The present study encapsulates the acute toxicological aspects of the Andrographis paniculata coupled with silver nanoparticles (AP-Ag NP). For acute toxicity studies according to OCED (Organization for Economic Cooperation and Development) guidelines Swiss Albino male mice (6-7 weeks) were used and were given single intraperitoneal dose of 2000 and $5000 \mathrm{mg} / \mathrm{kg}$ body weight of the AP-Ag nanoparticle and were observed for mortality and other side effects for 14 days. The individual components of the formulation, viz. silver oxide in surface modified nano form and at low dose, and Andrographis paniculata are both biocompatible materials. No changes were found for general appearance, behavior and body weight, thus concluding that the nanocomposite formulation does not have a single dose toxicity.
\end{abstract}

\section{Introduction}

Nanomedicine is the medical application of nanotechnology. The applications of nanomedicine has been far and widespread. The size of nanomaterials is similar to that of most biological molecules and structures; therefore, nanomaterials can be useful for both in vivo and in vitro biomedical research and applications. Thus far, the integration of nanomaterials with biology has led to the development of diagnostic devices, contrast agents, analytical tools, physical therapy applications, and drug delivery vehicles. Nanomedicine seeks to deliver a valuable set of research tools and clinically useful devices in the near future [1, 2].

In recent years silver nanoparticles are widely applied in consumer products, food technology and textiles/fabrics due to its unique chemical and biological properties [3]. Silver nanoparticles are the first and most widely commercialized nanomaterial in medical and healthcare sectors. AgNPs has been used in a range of biomedical applications, owing to their antibacterial activity [4-6], antifungal properties and antiviral properties [8-10].

Green synthesis is defined as the use of environmentally compatible materials such as bacteria, fungi and plants in the synthesis of nanoparticles [11]. Alternatively, synthesis from biologically derived extracts offers several advantages such as rapid synthesis, high yields and importantly, the lack of costly downstream processing required to produce the particles [12-14]. Hence, nanoparticle synthesis from plant extracts tentatively offers a route for large scale production of commercially attractive nanoparticles.

Toxicity testing is essential in the examination of newly developed drugs before it can be used on humans. It is the limitation of potential hazards a test substance may probably produce and the description of its action, most of the toxicity testing is done on experimental animals [15]. Toxicity studies are divided into, acute toxicity, sub-acute toxicity and chronic toxicity studies. Between the different types of toxicological studies, acute toxicity studies supply input about the overall profile of magnitude of a drug toxicity, its activity and overall effects [16]. One of the

*Corresponding Author:atischatterjee@gmail.com(Atiskumar Chattopadhyay) basic steps in toxicological estimation of a new substance is the account of its toxicity after a single exposure of that substance. So, current problem for nanomedicine involve understanding the issues related to toxicity and environmental impact of nanoscale materials.

Considering the vast potentiality of plants as sources this work aims to apply a biological green technique for the synthesis of silver nanoparticles as an alternative to conventional methods. In this regard, leaf extract of Andrographis paniculata (commonly known as Kalmegh) a species of family Acanthaceae was used for bioconversion of silver ions to nanoparticles. In this study, silver nanoparticles can be produced at low concentration of leaf extract without using any additional harmful chemical/physical methods. The effect of concentration of metal ions and concentration of leaf extract quantity were also evaluated to optimize route to synthesis silver nanoparticle. The method applied here is simple, cost effective, easy to perform and sustainable. Optical properties of the synthesized NPs were measured using UV-visible (UV-Vis) spectroscopy. Morphology of the prepared samples was analyzed by high resolution transmission electron microscopy (HRTEM), X-Ray Diffraction (XRD) were used to study the crystallinity of the sample. The present work evaluates the toxicity of the prepared samples and ensures their safety upon experimental mice model and also analyzes their behavioural changes if any upon their in vivo application.

\section{Experimental Methods}

Andrographis paniculata was collected from Jadavpur University campus. Swiss albino mice (6-7 days old) weighing $32 \pm 5$ g were procured from a CPCSEA registered animal house to conduct the toxicity study of the aforesaid nanoparticles.

\subsection{Synthesis of AP Conjugated Silver Nanoparticles (NPS)}

\subsubsection{Plant Extract Preparation}

About $1 \mathrm{~g}$ of AP leaves were washed thoroughly with plenty of distilled water and both surface of leaves were sterilized using alcohol by gently rubbing. These leaves were heated for $15 \mathrm{~min}$ in $50 \mathrm{~mL}$ of distilled water at $50^{\circ} \mathrm{C}$. Then the extract was filtrated with Whatman filter paper no 1 and 
further filtered using vacuum filter with pore size of $0.2 \mu \mathrm{m}$. The final filtrate was stored in cool dry place for further use.

\subsubsection{Green Synthesis of Silver Nanoparticles}

Initially, $50 \mathrm{mM}$ of silver nitrate $\left(\mathrm{AgNO}_{3}\right)$ solution was prepared. Then plant extract was added drop wise to it under stirring. The reaction mixture became yellowish to brownish and precipitate of silver was formed. The reaction mixture was left for $30 \mathrm{~min}$ for complete reduction to silver nitrate (Fig. 1). Then the precipitate was collected by centrifugation at $15000 \mathrm{rpm}$ for $10 \mathrm{~min}$ at $4{ }^{\circ} \mathrm{C}$. The precipitate was vacuum dried at $30{ }^{\circ} \mathrm{C}$ and the sample, i.e AP conjugated silver nanoparticle (AP-Ag NP) was stored for further studies.
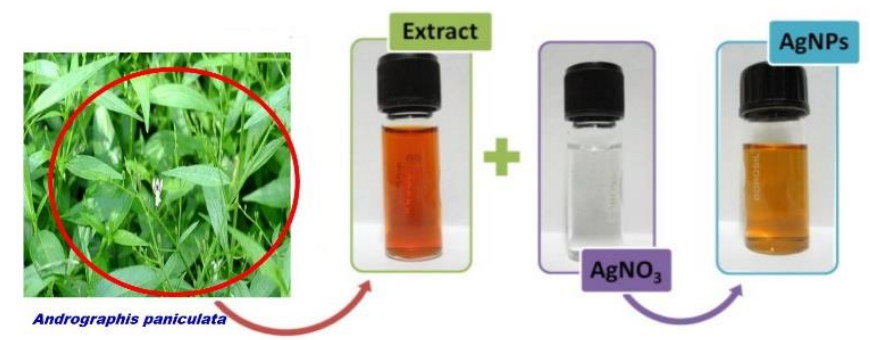

Fig. 1 Synthesis of AP-AgNPs

\subsection{Experimental Animals}

Swiss albino male mice of 6-7 weeks old and weighing approximately $(32 \pm 2.0 \mathrm{~g})$ were procured from a CPCSEA approved animal house (Registration No. 50/CPCSEA/1999) and randomly divided into three groups of six mice each $(n=6)$ and all of them received standard laboratory diet (Hindustan Lever, Kolkata) and water ad libitum. The animals were housed in large clean polypropylene cages in a temperaturecontrolled room $\left(20 \pm 2{ }^{\circ} \mathrm{C}\right)$ with relative humidity (45-60\%) under $12 \mathrm{~h}$ light and dark cycles during the whole study period. Prior to experimentation, acclimatization was done for 7 days. The animals were maintained according to the guidelines recommended by Committee for the Purpose of Control and Supervision of Experiments on Animals (CPCSEA), New Delhi, India and approved by the Institutional Animal Ethics Committee (IAEC) (Approval No. IAEC/PHARM/1503/03/2015 dated 30.11.15). All procedures complied with the Declaration of Helsinki, as revised in 1996.

\subsection{Characterization of Synthesized AP-Ag NP}

UV-Vis spectra of the aqueous solutions of synthesized Ag NPs were recorded in $\lambda 25$ spectrophotometer (Perkin Elmer, Germany) within the range of 200 to $800 \mathrm{~nm}$. XRD patterns of the synthesized materials were analyzed in the range of $2 \theta$ from $25^{\circ}$ to $80^{\circ}$ using powder diffractometer, Model D8, BRUKER AXS, using $\mathrm{Cu} \mathrm{K} \alpha$ radiation ( $\alpha=0.15425 \mathrm{~nm}$ ). For this purpose, solution of Ag NPs was repeatedly drop casted on glass slides to make a thick coating of Ag NPs. Surface morphology and shape distribution was studies with the help of HRTEM.

\subsection{Appraisal of Acute Toxicity}

Acute toxicity studies of the nanoconjugate was carried out in mice by using Organisation for Economic Co-operation and Development (OECD) guideline 425 [9]. Before administration of a single dose of the test samples, the mice were deprived of food for $3 \mathrm{~h}$. Doses of 2000 and 5000 $\mathrm{mg} / \mathrm{kg}$ of the test samples were given to Group II and Group III respectively, whereas Group I served as vehicle control and were orally administered $2 \mathrm{~mL}$ of distilled water. The respective doses were suspended in water and were administered orally.

The mice were observed after every $30 \mathrm{~min}$ post nanocomposite treatment for $2 \mathrm{hrs}$. Once daily cage side observations included changes in skin, fur, eyes, mucous membrane (nasal), autonomic (salivation, lacrimation, perspiration, piloerection, urinary incontinence, and defecation) and central nervous system (drowsiness, gait, tremors and convulsion) changes. Mortality, if any, was determined over a period of 2 weeks. After completion of the treatment, the animals were sacrificed by cervical dislocation and necropsied to facilitate gross pathological examination of organs. Surface morphology and shape distribution was studies with the help of HRTEM.

\subsection{Clinical Observation}

The treated animals were observed for mortality (twice daily) and the clinical signs were recorded to note the onset, duration and reversal (if any) of toxic effect at 2, 4, 6 and 8 hours after the administration of last https://doi.org/10.30799/jnst.175.19050111 substances and once daily thereafter for 14 days. The routine cage side observation s included changes in skin and fur, eye and mucus membrane, somatomotor activity, general behavior pattern were noted. Miscellaneous sings like arching of the back, alopecia, wound, nasal discharge, lacrimation and loose stool were also recorded during the observation.

\section{Results and Discussion}

\subsection{Characterization of NPS}

The UV-Vis spectra of both chemically synthesized and green synthesized nanomaterials (Fig. 2) showed absorption band-between wavelength range $200-800 \mathrm{~nm}$, with a peak at $360 \mathrm{~nm}$, conforming presence of Ag NPs in the solution. In case of AP conjugated AgNPs (APAgNps) the absorption spectra remained almost same with a slight blue shift in the absorption maxima. The XRD pattern of the synthesized nanomaterial (Fig. 2b) showed diffraction peaks at $2 \theta=36.2^{\circ}, 40.6^{\circ}, 62.6^{\circ}$ and $71.3^{\circ}$, which respectively corresponded to (111), (200), (220) and (311) planes of pure silver. All peaks were duly assigned by using JCPDS file no. 04-0783 pertaining to pure silver. HRTEM micrograph confirmed well distribution spherical particles of the synthesized NP.

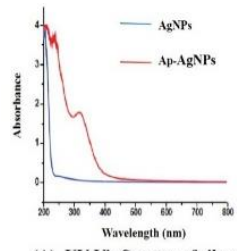

(A). UV-Vis Spectra of silver nanoparticles of $A$. paniculata

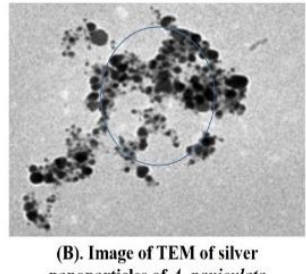

(B). Image of TEM of silver
nanoparticles of $A$. paniculata

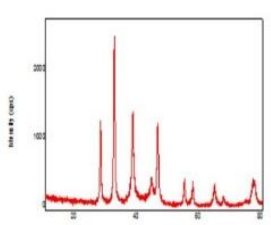

(C). 5 XRD of silver nanoparticles of $A$. paniculata
Fig. 2 Characterization of silver nanoparticles (AgNPs)

\subsection{Behavioural Observations and General Appearance}

The behavioral parameters of animals were observed 2,4,6 and $8 \mathrm{~h}$ after the administration of the nanocomposite according to the standard protocol. No marked changes were observed in the wellness parameters observed. There were no mortality and all the mice survived the 14 day study period.

\subsection{Body Weight, Daily Food Consumption and Daily Water Intake}

Tables 1 and 2 shows the change observed before and after administration of the nanocomposite, there were very slight increase in body weight of the animals but were statistically insignificant (Fig. 3). Daily food consumption and daily water intake pattern were almost normal in comparison to normal control animals.

Table 1 Effect of AP-AgNPs on the body weight of mice at 2,000 mg/kg dose

\begin{tabular}{llll}
\hline Animals (Mice) & 0 Day & $7^{\text {th }}$ day & $14^{\text {th }}$ day \\
\hline Control & $24.3 \pm 2.66$ & $27.5 \pm 2.07$ & $30.2 \pm 0.99$ \\
$2000 \mathrm{mg} / \mathrm{kg}$ body wt & $24.4 \pm 1.35$ & $25.4 \pm 2.24$ & $27.9 \pm 1.22$ \\
$5000 \mathrm{mg} / \mathrm{kg}$ body wt. & $26.7 \pm 0.89$ & $25.9 \pm 1.34$ & $27.6 \pm 1.54$ \\
\hline
\end{tabular}

Table 2 Effect of AP-AgNPs on the body weight of mice at 5,000 mg/ kg dose

\begin{tabular}{llll}
\hline Animals (Mice) & 0 Day & $7^{\text {th }}$ day & $14^{\text {th }}$ day \\
\hline Control & $24.2 \pm 2.15$ & $27.1 \pm 1.65$ & $30.6 \pm 1.02$ \\
$2000 \mathrm{mg} / \mathrm{kg}$ body wt & $24.5 \pm 1.55$ & $26.5 \pm 1.93$ & $28.2 \pm 1.27$ \\
$5000 \mathrm{mg} / \mathrm{kg}$ body wt. & $24.1 \pm 0.98$ & $25.8 \pm 1.05$ & $27.1 \pm 1.06$ \\
\hline
\end{tabular}
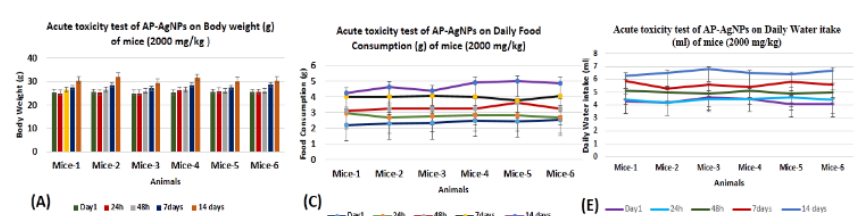

(c)

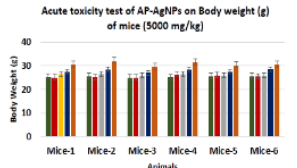

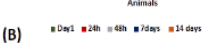
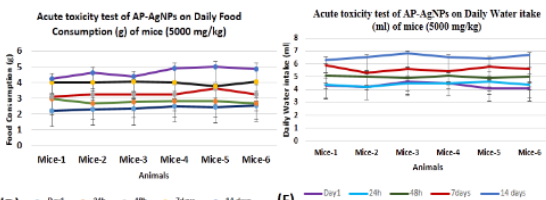

Fig. 3 Effect of AP-AgNPs on body weight, food consumption and water intake 


\subsection{Behavioral Observations and General Appearance}

In this study the behavioral parameters and appearance of animals after drug administration is indicator of the toxicity of the test drug. The behavioral patterns of animals were observed in $2 \mathrm{~h}, 4 \mathrm{~h}, 6 \mathrm{~h}$ and $8 \mathrm{~h}$ interval and followed by $14 \mathrm{~h}$ after the administration. The behavioral parameters and appearance was observed according to the standard protocol. No significant changes were observed in wellness parameters used for evaluation of toxicity. Skin, fur, eyes, mucous membrane, behavioral pattern, salivation and sleep pattern parameters of the treated animals were found to be normal (Tables 3 and 4). No toxic symptom or mortality was observed in any mice. All treated mice lived up to 14 days after the administration of NPs.

Table 3 Clinical observations of mice at 2,000 mg/kg dose of AP-AgNPs

\begin{tabular}{|c|c|c|c|c|c|c|}
\hline Signs and symptoms & Mice 1 & Mice 2 & Mice 3 & Mice 4 & Mice 5 & Mice 6 \\
\hline Behavior & Normal & Normal & Normal & Normal & Normal & Normal \\
\hline Somatomotor activity & Normal & Normal & Normal & Normal & Normal & Normal \\
\hline Skin and Fur & Normal & Normal & Normal & Normal & Normal & Normal \\
\hline $\begin{array}{l}\text { Eyes and mucous } \\
\text { membranes }\end{array}$ & Normal & Normal & Normal & Normal & Normal & Normal \\
\hline Salivation & Absent & Absent & Absent & Absent & Absent & Absent \\
\hline Diarrhoea & Absent & Absent & Absent & Absent & Absent & Absent \\
\hline Tremors/ convulsions & Absent & Absent & Absent & Absent & Absent & Absent \\
\hline Death & $\mathrm{Nil}$ & $\mathrm{Nil}$ & $\mathrm{Nil}$ & Nil & $\mathrm{Nil}$ & $\mathrm{Nil}$ \\
\hline Other symptoms & Nil & $\mathrm{Nil}$ & $\mathrm{Nil}$ & Nil & $\mathrm{Nil}$ & Nil \\
\hline
\end{tabular}

Table 4 Clinical observations of mice at 5,000 mg/kg dose of AP-AgNPs

\begin{tabular}{lllllll}
\hline Signs and symptoms & Mice 1 & Mice 2 & Mice 3 & Mice 4 & Mice 5 & Mice 6 \\
\hline Behavior & Normal & Normal & Normal & Normal & Normal & Normal \\
Somatomotor activity & Normal & Normal & Normal & Normal & Normal & Normal \\
Skin and Fur & Normal & Normal & Normal & Normal & Normal & Normal \\
$\begin{array}{l}\text { Eyes and mucous } \\
\text { membranes }\end{array}$ & Normal & Normal & Normal & Normal & Normal & Normal \\
Salivation & & & & & & \\
Diarrhoea & Absent & Absent & Absent & Absent & Absent & Absent \\
Tremors/ convulsions & Absent & Absent & Absent & Absent & Absent & Absent \\
Death & Absent & Absent & Absent & Absent & Absent & Absent \\
Other symptoms & Nil & Nil & Nil & Nil & Nil & Nil \\
& Nil & Nil & Nil & Nil & Nil & Nil \\
\hline & & & & & &
\end{tabular}

Table 5 Effect of AP-AgNPs on the Necropsy of mice at 2,000 mg/kg dose

\begin{tabular}{lll}
\hline Experimental Animals & $\begin{array}{l}\text { Observed lesions during } \\
\text { study }(2000 \mathrm{mg} / \mathrm{kg})\end{array}$ & $\begin{array}{l}\text { Observed lesions during } \\
\text { study }(5000 \mathrm{mg} / \mathrm{kg})\end{array}$ \\
\hline 1. & Nil & Nil \\
2. & Nil & Nil \\
3. & Nil & Nil \\
4. & Nil & Nil \\
5. & Nil & Nil \\
6. & Nil & Nil \\
\hline
\end{tabular}

\subsection{Necropsy}

All the animals were sacrificed on day 14 and necropsied. Body cavities (cranial, thoracic, abdominal and pelvic) did not reveal any lesions nor any inflammation or any abnormalities (Table 5).

\section{Conclusion}

There was no change in the general health of the animals throughout the study. The results indicate that the nanocomposite did not exhibit any toxicity. These findings may facilitate the development of safe and efficient Ag-AP nanoconjugate as an effective therapy against various health disorders.

\section{References}

[1] V. Wagner, A. Dullaart, A.K. Bock, A. Zweck, The emerging nanomedicine landscape, Nature Biotech. 24(10) (2006) 1211-1217.

[2] R.A. Freitas, What is nanomedicine?, Nanomed. 1(1) (2005) 2-9.

[3] D.R. Monteiro, L.F. Gorup, A.S. Takamiya, A.C. Ruvollo-Filho, E.R. de Camargo, The growing importance of materials that prevent microbial adhesion: antimicrobial effect of medical devices containing silver, Int. J. Antimicrob. 34 (2009) 103-110.

[4] X. Chen, H.J. Schluesener, Nanosilver: a nanoproduct in medical application, Toxicol. Lett. 176 (2008) 1-12.

[5] 0. Salata, Application of nanoparticles in biology and medicine, J. Nanobiotechnol. 2 (2004) 1-6.

[6] J.B. Wright, K. Lam, D. Hansen, R.E. Burrell, Efficacy of topical silver against fungal burn wound pathogens, Am. J. Infect. Control. 27 (1999) 344-350.

[7] J. Chen, M.K. Choe, S. Chen, S. Zhang, Community environment and HIV/AIDSrelated stigma in China, AIDS Educ Prev. 17 (2005) 1-11.

[8] J.L. Elechiguerra, J.L. Burt, J.R. Morones, A. Camacho-Bragado, X. Gao, H.H. Lara, Interaction of silver nanoparticles with HIV-1, J. Nanobiotech. 3(6) (2005) 110.

[9] P.V. Asharani, M.P. Hande, S. Valiyaveettil, Anti-proliferative activity of silver nanoparticles, BMC Cell Biol. 10(65) (2009) 1-14.

[10] V. Ernest, C. George, Priya Doss, A. Muthiah, A. Mukherjee, N. Chandrasekaran, Genotoxicity assessment of low concentration AgNPs to human peripheral blood lymphocytes, Int. J. Pharm. Pharm. Sci. 5 (2013) 377-381.

[11] J.K. Patra, K.H. Baek, Green nanobiotechnology: factors affecting synthesis and characterization techniques, J. Nanomater. 2014 (2014) 417305-1-12.

[12] R. Gannimani, A. Perumal, S. Krishna, A. Mishra, P. Govender, Synthesis and antimicrobial activity of silver and gold nanoparticles produced using aqueous seed extract of Protorhus longifolia as reducing agent, Dig. J. Nanomater. Biostruct. 9 (2014) 1669-1679.

[13] V.L. Das, R. Thomas, R.T. Varghese, E. Soniya, J. Mathew, E. Radhakrishnan Extracellular synthesis of silver nanoparticles by the Bacillus strain CS 11 isolated from industrialized area, Biotech. 4 (2014) 121-126.

[14] B. Liu, J. Xie, J. Lee, Y. Ting, J.P. Chen, Optimization of high-yield biological synthesis of single-crystalline gold nanoplates, J. Phys. Chem. B 109 (2005) 15256-15263.

[15] H. Cunny, E. Hodgson, A test book on modern toxicity, $3^{\text {rd }}$ Ed., A John Wiley and Son, Inc., Hoboken, NJ, USA. 2004, pp.353-484.

[16] C.J. Lee, L.H. Lee, CH. Lu, Development and evaluation of drugs from laboratory through licensure to market, 2nd Ed., CRC Press, USA, 2003. 